\title{
Torsade de Pointes due to Methadone Use in a Patient with HIV and Hepatitis C Coinfection
}

\author{
Jinu John, ${ }^{1}$ Xixi Amley, ${ }^{2}$ Gabriel Bombino, ${ }^{2}$ Chaim Gitelis, ${ }^{2}$ Bernard Topi, ${ }^{1}$ \\ Gerald Hollander, ${ }^{2}$ and Joydeep Ghosh ${ }^{2}$ \\ ${ }^{1}$ Department of Internal Medicine, Maimonides Medical Center, 4802 Tenth Avenue, Brooklyn, NY 11219, USA \\ ${ }^{2}$ Division of Cardiology, Maimonides Medical Center, 4802 Tenth Avenue, Brooklyn, NY 11219, USA \\ Correspondence should be addressed to Jinu John, jjohn3@maimonidesmed.org
}

Received 15 October 2010; Revised 2 December 2010; Accepted 9 December 2010

Academic Editor: Brian Olshansky

Copyright (C) 2010 Jinu John et al. This is an open access article distributed under the Creative Commons Attribution License, which permits unrestricted use, distribution, and reproduction in any medium, provided the original work is properly cited.

We present a case of Torsade de Pointes secondary to multiple factors including patient susceptibility and iatrogenic influences. Contributing causes are presented, and the approach to treatment is discussed.

\section{Case Report}

A 50-year-old male with a past history of HIV infection, acquired immune deficiency syndrome (AIDS), pneumocystis carinii pneumonia, hepatitis $\mathrm{C}$, and hepatoma (postradiofrequency ablation) presented to the emergency room for weakness and syncope which occurred in the bathroom while straining to defecate. He denied palpitation, chest pain, or shortness of breath prior to losing consciousness. There was no history of seizure disorder. The following day while walking to the hospital, he developed lightheadedness again which was associated with diaphoresis and had to hold onto a pole for support for two minutes before feeling able to continue. There was a history of cocaine and IV heroin abuse (discontinued two years ago).

Prehospital medications included lamivudine/zidovudine, atazanavir, trimethoprim/sulfamethoxazole, and methadone. He was on antiretroviral drugs for 10 years, and the methadone dosage was increased to $40 \mathrm{mg} /$ day two months prior to this presentation. On admission in the ER, he had a regularly irregular pulse and a blood pressure of 124/60. Physical examination was unremarkable except for ecchymosis in the left periorbital region. Relevant lab reports produced the following results: MCV $131 \mathrm{FL}$, platelet count $82,000 / \mathrm{mm}^{3}$, AST $176 \mathrm{IU} / \mathrm{L}$, alkaline phosphatase $140 \mathrm{IU} / \mathrm{L}$, ALT $104 \mathrm{IU} / \mathrm{L}$, albumin $3.2 \mathrm{~g} / \mathrm{dL}$, and INR 1.3. Serum potassium, magnesium, corrected calcium, and cardiac enzymes were within normal range. The patient's chest X-ray was unremarkable. EKG recorded 14 months prior to the ER presentation showed sinus rhythm and QTc $455 \mathrm{~ms}$ (Figure 1). The initial EKG on presentation showed ventricular bigeminy (Figure 2) with QTc $550 \mathrm{~ms}$. In the ER, he developed two episodes of TdP (Figure 3 ) each resolving spontaneously, although the patient became unconscious for approximately two minutes during each episode. He was given $2 \mathrm{~g}$ Magnesium IV and Amiodarone IV push $150 \mathrm{mg}$ twice. The patient was transferred to cardiac ICU. HIV medications were switched to raltegravir, emtricitabine/tenofovir; methadone was discontinued. Three days later, he developed sinus bradycardia at 38 beats per min (bpm) with QTc $571 \mathrm{~ms}$ (Figure 4). To shorten his QTc and prevent further episodes of TdP, the patient underwent a temporary pacemaker insertion and was paced at a rate of $100 \mathrm{bpm}$ (Figure 5). After two days, his QTc slowly decreased and sinus bradycardia resolved. 23 days following the modification of the medications administered to the patient, the EKG still exhibited prolonged QTc (Figure 6). As the patient was considered at high risk to develop TdP, he underwent an implantable cardioverter defibrillator (ICD) placement. He is currently being followed with no further episodes of TdP.

\section{Discussion}

TdP belongs to a group of polymorphic ventricular tachycardia characterized by varying QRS amplitudes that appear 


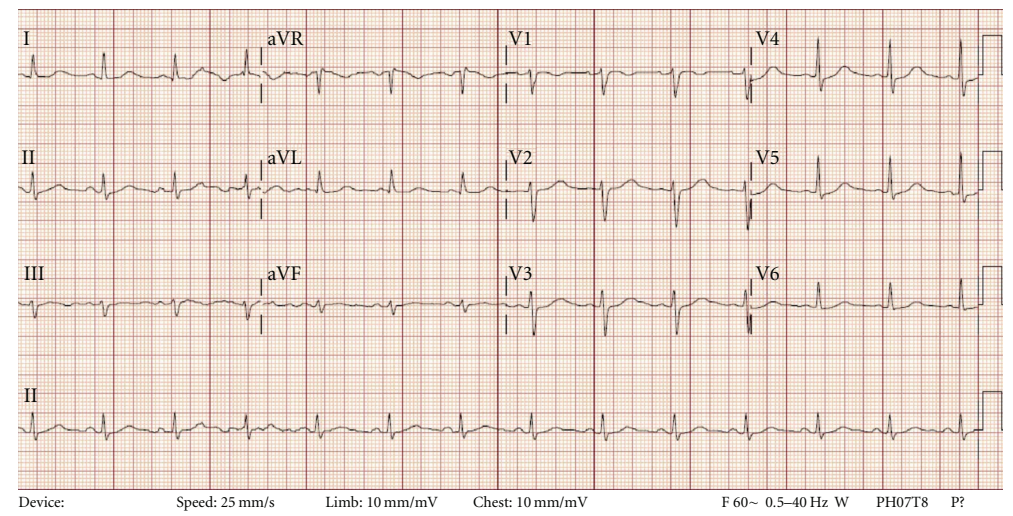

FIGURE 1: Baseline EKG was acquired about 14 months prior to the syncope episodes. QTc 455 ms.

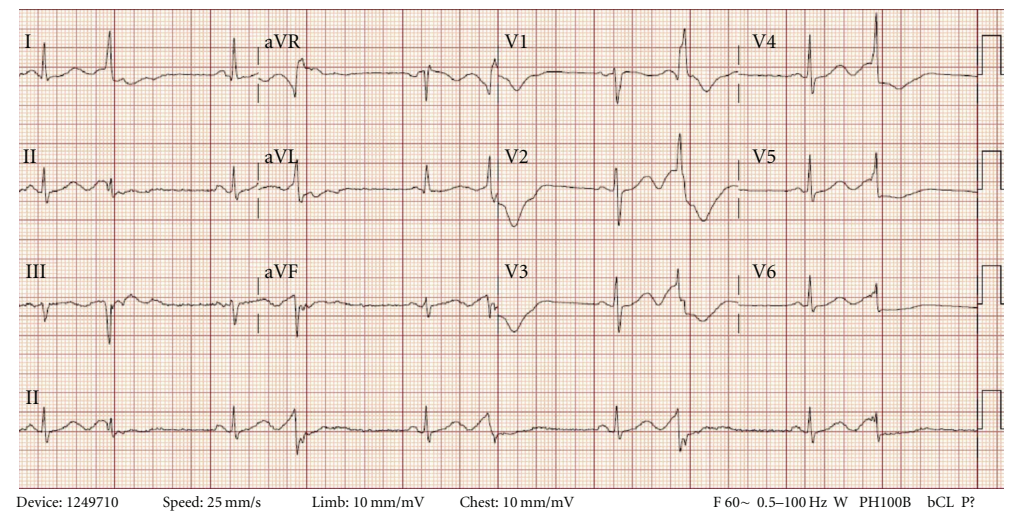

FIgURE 2: Initial 12 lead EKG in emergency room showing ventricular bigeminy. QTc was 550 ms.

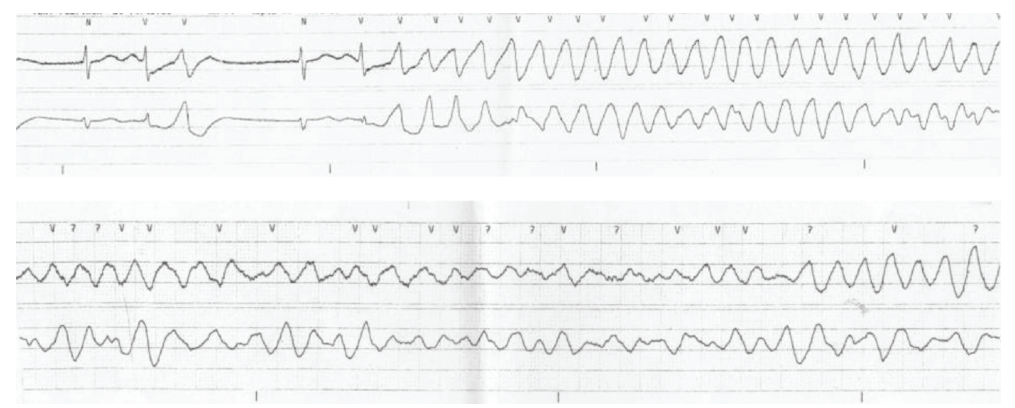

FIGURE 3: EKG tracing in emergency room showed short-long-short sequence of RR cycle followed by TdP. The patient had two episodes of syncope while he had TdP. The above two tracings are from one of the two episodes.

to twist around an isoelectric line in the setting of long QTc as revealed in this patient's baseline EKG. TdP is commonly preceded by long-short R-R cycle which is followed by a late premature ventricular complex. Often, early after depolarization manifested as T-U waves in EKG precede TdP. In patients with prolonged QTc, the presence of pathological $\mathrm{T}-\mathrm{U}$ waves (Figure 6) might be the only warning sign of imminent TdP [1].

Very often, the cause of TdP is multifactorial. The common risk factors of TdP include (a) female gender, (b) heart disease, (c) electrolyte abnormalities, (d) bradyarrhythmias, (e) stroke, and (f) a significant universe of drugs that lead to QT prolongation [2-4]. The upper limit of the duration of QTc is often considered to be $430 \mathrm{~ms}$ for adult males and $450 \mathrm{~ms}$ for adult females, with a normal range of plus or minus 15 percent. QTc prolongation is defined as a QTc of more than $450 \mathrm{~ms}$ for adult males and $470 \mathrm{~ms}$ for adult females [5]. Studies have shown that QTc prolongation is an independent predictor of cardiovascular mortality, especially from ventricular arrhythmias such as TdP [6].

There is an increased prevalence of QTc prolongation in patients with active HIV infection. Sani and Okeahialam 


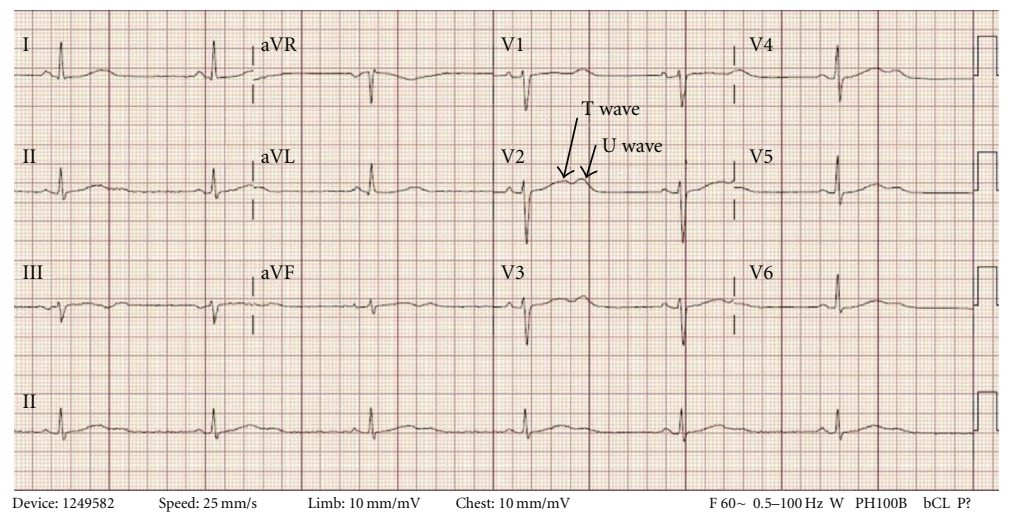

FIGURE 4: Sinus bradycardia with heart rate of 38 beats per minute occurred three days after admission. QTc 571 ms. Also noted are pathological T-U waves.

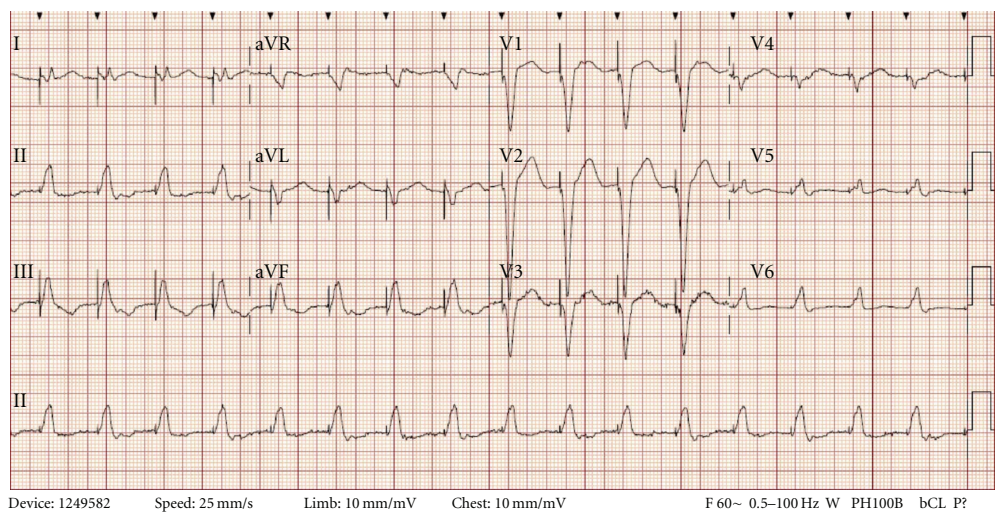

FIGURE 5: Intravenous temporary pace maker was inserted for sinus bradycardia with long QTc. To prevent TdP, pacing rate was set at 100 beats per minute.

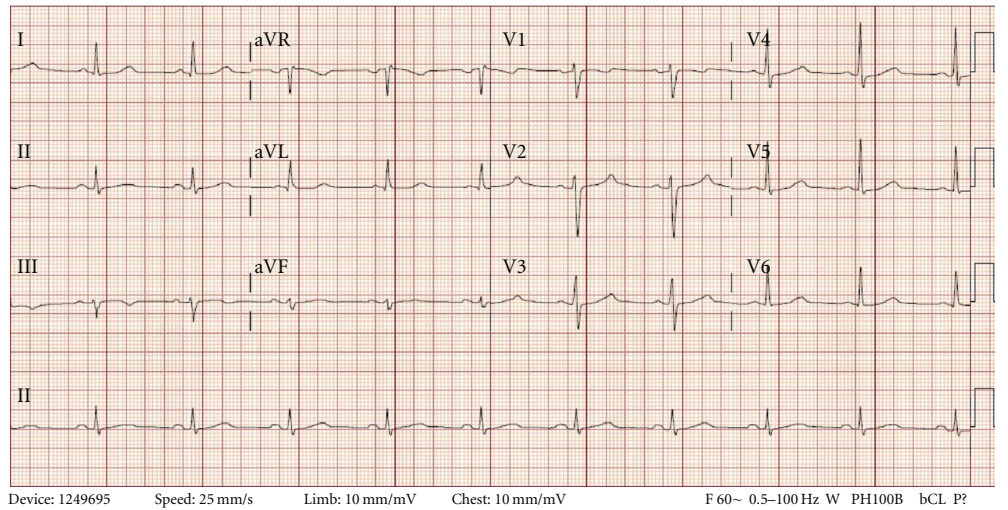

FIGURE 6: 23 days after admission, the EKG showed a QTc of $492 \mathrm{~ms}$ prior to ICD placement. The pathological T-U waves are no longer seen.

showed that prolonged QTc was present in $28 \%$ of such patients. In patients with AIDS, the prevalence increased to $45 \%$ [7]. In patients with HIV and autonomic neuropathy, the prevalence of QTc prolongation is reported to be 65\% [8]. The exact reason for such correlation between QTc prolongation and HIV infection is still a topic of debate. But chronic HIV infection itself and many drugs used to treat HIV and other opportunistic infections are plausible explanations. In contrast, Charbit et al. suggested that QTc prolongation is related to the duration of a patient's HIV infection rather than medications used during the treatment of HIV or associated opportunistic infections [9]. Protease inhibitors (PIs) are a group of medications implicated in QTc prolongation because of the in vitro inhibition of 
the hERG (human ether-a-go-go-related gene) current [10]. But Charbit et al. concluded that protease inhibitors are not independently associated with QTc prolongation [9].

Methadone is a very frequently used drug in the management of pain and heroin addiction. Methadone causes the blockade of hERG current and causes clinically significant QTc prolongation. Martell et al. found a $10.8 \mathrm{~ms}$ increase in QTc within 2 months of starting methadone in a group of heroin users [11]. The prevalence of methadone-induced QTc prolongation ranges between $16 \%$ and 33\%, and this can happen within a wide dose range of methadone (29$1690 \mathrm{mg} /$ day) [12]. But methadone doses less than $40 \mathrm{mg} /$ day do not commonly cause QTC prolongation [13]. QTc prolongation caused by methadone is dose dependant and often recent dose escalation is associated with TdP. Methadone is metabolized by CYP3A4, which is also induced by PIs. Patients, when started on PIs, might require escalation of the methadone dose for symptom control which can further prolong QTc. Methadone is also known to cause appearance of pathological $U$ waves in EKG which may be an indicator of imminent TdP in some cases [14].

Hepatitis $\mathrm{C}$ is indicated as an independent risk factor for QTc prolongation. Nordin et al. investigated the impact of Hepatitis C and HIV coinfection on QTc prolongation and suggested that hepatitis $\mathrm{C}$ co-infection nearly doubled the risk of QTc prolongation in HIV positive patients. Liver cirrhosis, low albumin, and high AST predict QTc prolongation. These three factors tend to cluster with Hepatitis $\mathrm{C}$ which explains the increased risk of QTc prolongation associated with Hepatitis C infection [15].

The above case shows how multiple contributing factors can cumulatively cause TdP in patients who already are at risk due to genetic predisposition. This patient had a long QTc to start with and also had HIV and Hepatitis C/liver disease. He also was on antiretroviral medications for 10 years. Methadone dose escalation might have prolonged the QTc further and caused appearance of T-U waves. In this setting, a critically timed premature complex triggered off TdP. As the patient had multiple risk factors and continued to have an abnormal QTc despite changing medications, he was considered to be at high risk for further development of TdP, and the decision of utilizing an ICD was made.

This case also highlights the need for carefully monitoring susceptible patients treated with methadone for potentially dangerous QT prolongation which can occur even after the patient has been on therapy for some time. Patients should also be made aware of relevant drug interactions and the importance of reporting episodes of dizziness or near syncope. Recent guidelines suggest getting a baseline EKG before starting patients on methadone. After initiation of therapy, EKG should be repeated to look for QTc prolongation within one month and then annually even if patients are asymptomatic. Discontinuation of methadone should be considered if QTc > $500 \mathrm{~ms}$ [16].

\section{References}

[1] P. Kirchhof, M. R. Franz, A. Bardai, and A. M. Wilde, "Giant $\mathrm{T}-\mathrm{U}$ waves precede torsades de pointes in long QT syndrome: a systematic electrocardiographic analysis in patients with acquired and congenital QT prolongation," Journal of the American College of Cardiology, vol. 54, no. 2, pp. 143-149, 2009.

[2] A. G. Kocheril, S. A. J. Bokhari, W. P. Batsford, and A. J. Sinusas, "Long QT(c) and torsades de pointes in human immunodeficiency virus disease," Pacing and Clinical Electrophysiology, vol. 20, no. 11, pp. 2810-2816, 1997.

[3] D. M. Roden, "Taking the "idio" out of "idiosyncratic": predicting torsades de pointes," Pacing and Clinical Electrophysiology, vol. 21, no. 5, pp. 1029-1034, 1998.

[4] Arizona Center for Education and Research on Therapeutics. University of Arizona College of Pharmacy. Resources for Professionals, "Torsades List: Drugs With a Risk of Torsades de Pointes," http://www.azcert.org/medical-pros/druglists/bycategory.cfm.

[5] I. Goldenberg, A. J. Moss, and W. Zareba, "QT interval: how to measure it and what is "normal"', Journal of Cardiovascular Electrophysiology, vol. 17, no. 3, pp. 333-336, 2006.

[6] E. G. Schouten, J. M. Dekker, P. Meppelink, F. J. Kok, J. P. Vandenbroucke, and J. Pool, "QT interval prolongation predicts cardiovascular mortality in an apparently healthy population,” Circulation, vol. 84, no. 4, pp. 1516-1523, 1991.

[7] M. U. Sani and B. N. Okeahialam, "QTc interval prolongation in patients with HIV and AIDS," Journal of the National Medical Association, vol. 97, no. 12, pp. 1657-1661, 2005.

[8] A. Villa, V. Foresti, and F. Confalonieri, "Autonomic neuropathy and prolongation of QT interval in human immunodeficiency virus infection," Clinical Autonomic Research, vol. 5, no. 1, pp. 48-52, 1995.

[9] B. Charbit, A. Rosier, D. Bollens et al., "Relationship between HIV protease inhibitors and QTc interval duration in HIVinfected patients: a cross-sectional study," British Journal of Clinical Pharmacology, vol. 67, no. 1, pp. 76-82, 2009.

[10] B. D. Anson, J. G. R. Weaver, M. J. Ackerman et al., "Blockade of HERG channels by HIV protease inhibitors," Lancet, vol. 365, no. 9460, pp. 682-686, 2005.

[11] B. A. Martell, J. H. Arnsten, M. J. Krantz, and M. N. Gourevitch, "Impact of methadone treatment on cardiac repolarization and conduction in opioid users," American Journal of Cardiology, vol. 95, no. 7, pp. 915-918, 2005.

[12] D. W. Abramson, D. K. Quinn, and T. A. Stern, "Methadoneassociated QTc prolongation: a case report and review of the literature," Primary Care Companion to the Journal of Clinical Psychiatry, vol. 10, no. 6, pp. 470-476, 2008.

[13] G. B. Ehret, C. Voide, M. Gex-Fabry et al., "Drug-induced long QT syndrome in injection drug users receiving methadone: high frequency in hospitalized patients and risk factors," Archives of Internal Medicine, vol. 166, no. 12, pp. 1280-1287, 2006.

[14] P. Athanasos, A. L. Farquharson, P. Compton, P. Psaltis, and J. Hay, "Electrocardiogram characteristics of methadone and buprenorphine maintained subjects," Journal of Addictive Diseases, vol. 27, no. 3, pp. 31-35, 2008.

[15] C. Nordin, A. Kohli, S. Beca et al., "Importance of hepatitis C coinfection in the development of QT prolongation in HIVinfected patients," Journal of Electrocardiology, vol. 39, no. 2, pp. 199-205, 2006.

[16] M. J. Krantz, J. Martin, B. Stimmel, D. Mehta, and M. C. P. Haigney, "QTc interval screening in methadone treatment," Annals of Internal Medicine, vol. 150, no. 6, pp. 387-395, 2009. 


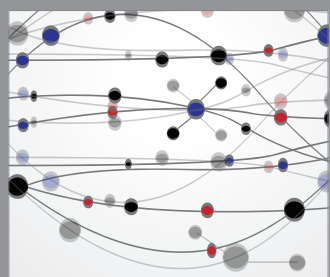

The Scientific World Journal
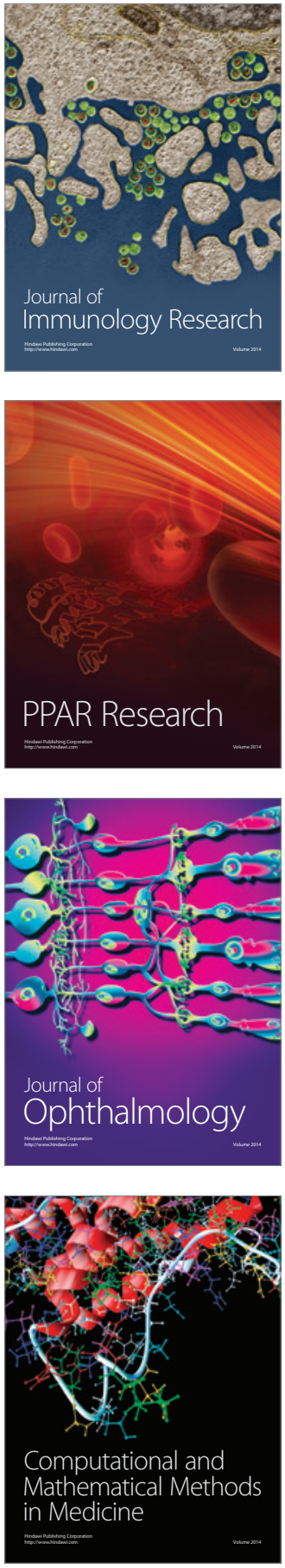

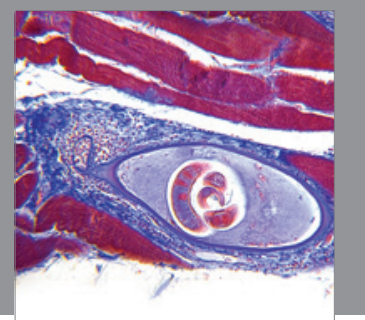

Gastroenterology

Research and Practice
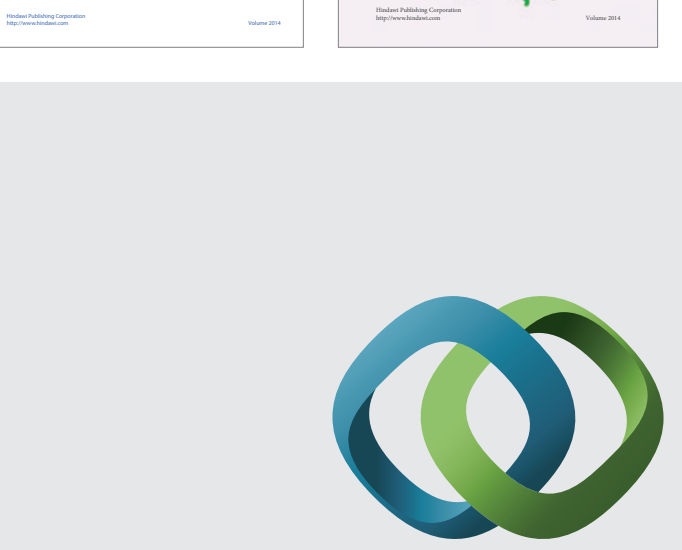

\section{Hindawi}

Submit your manuscripts at

http://www.hindawi.com
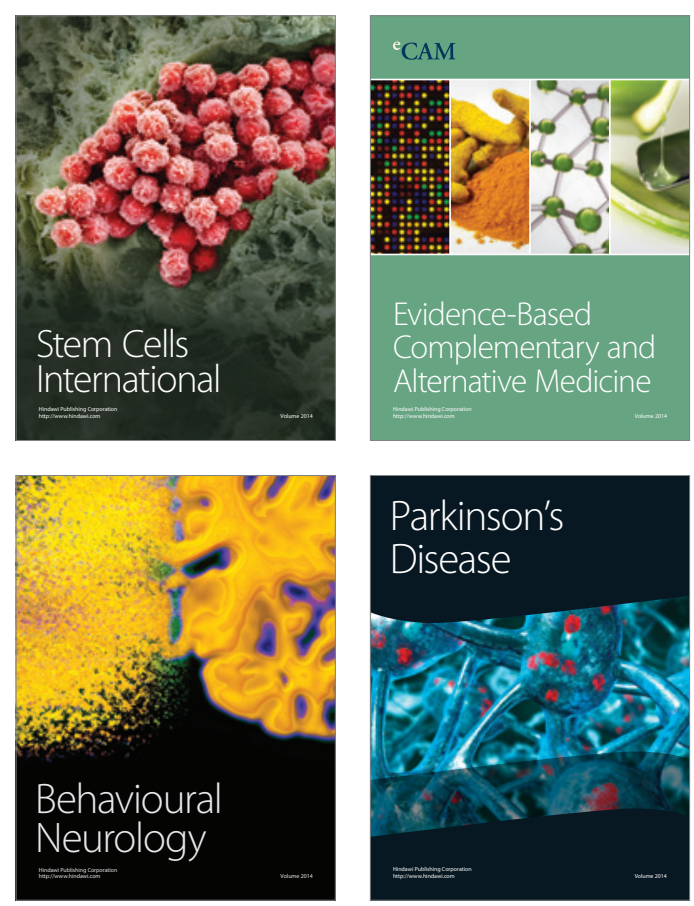

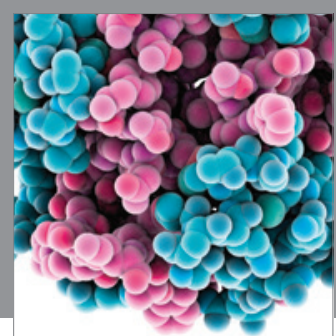

Journal of
Diabetes Research

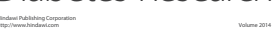

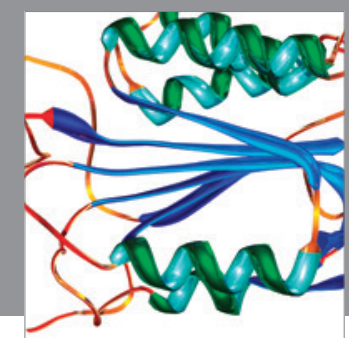

Disease Markers
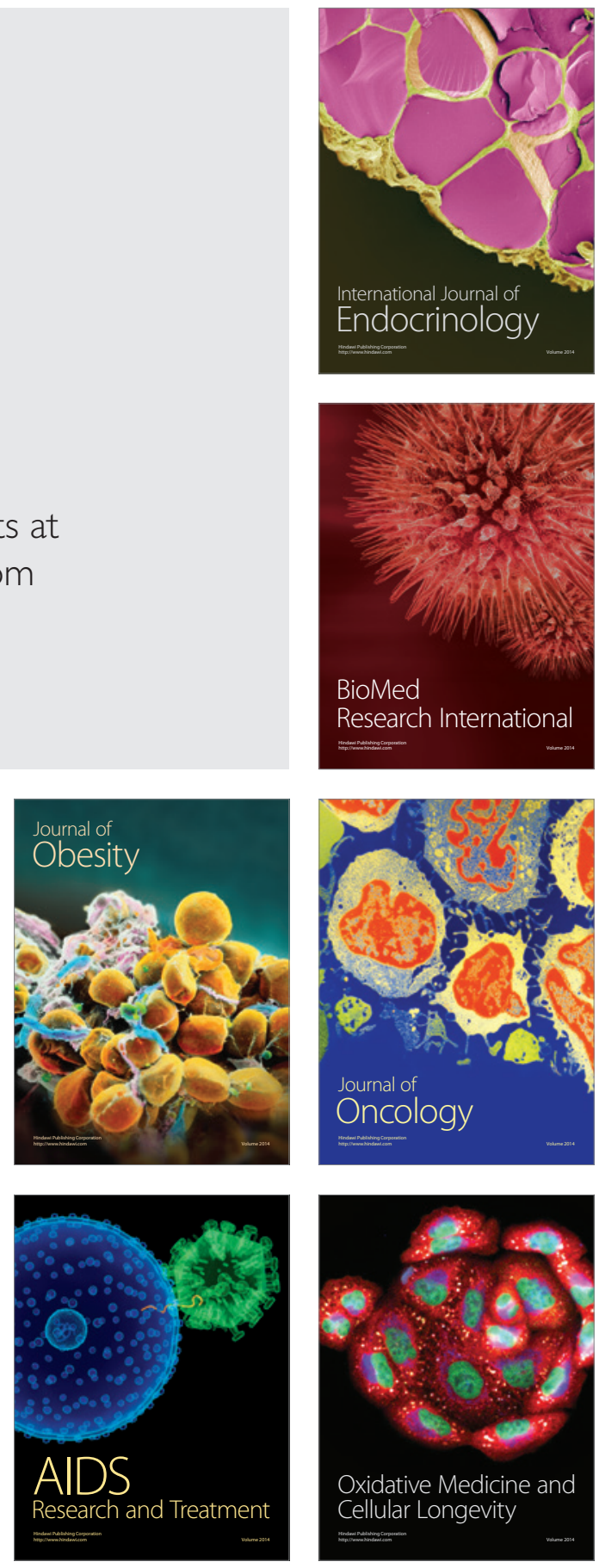